\title{
Multi-Cell User-Scheduling and Random Beamforming Strategies For Downlink Wireless Communications
}

\author{
Xiaojun Tang, Sean A. Ramprashad, and Haralabos Papadopoulos \\ DoCoMo Communications Labs USA, Inc., Palo Alto, CA 94304 \\ Email: xtang@winlab.rutgers.edu, \{ramprashad,hpapadopoulos\}@docomolabs-usa.com
}

\begin{abstract}
We consider random beamforming and user scheduling strategies in a multi-cell environment for downlink wireless communications. Our focus is on using combined scheduling and beamforming to mitigate performance losses due to inter-cell interference. Of interest are strategies requiring minimal intra-cell and inter-cell system information exchange. Indeed the acquisition and inter-cell exchange of such information can be significant overheads in a practical deployment.

Random beamforming requires substantially less channel state information than linear zero forcing strategies to schedule transmissions. It is therefore of practical interest despite some limitations in controlling intra-cell interference. We consider how one can use a minimal exchange of information, in a multi-cell multistep pattern of exchanges, to improve the overall performance of random beamforming in a multi-cell environment.
\end{abstract}

\section{INTRODUCTION}

We consider joint beamforming and user-scheduling in a multi-cell environment. The focus is on leveraging the combination of the two techniques to mitigate impairments due to inter-cell interference. Here two issues are of particular interest: 1) The amount and type of information obtained from users and shared between cells in order to schedule transmissions; and 2) How strategies can use such information to control inter-cell interference to improve system performance.

The problem of inter-cell interference (ICI) in Multiple Input Multiple Output (MIMO) transmission deployments in a multi-cell environment is one that has been receiving recent attention in both standards and research. It is well understood that an obstacle limiting the performance of MIMO and its increased spectral efficiency [1]-[3] is that of inter-cell interference between MIMO transmissions [1]. While many existing strategies can be combined with MIMO to manage such interference, e.g. use of a classic frequency reuse pattern, MIMO brings in the use of the "space" dimension to control inter-cell interference. This space dimension can be leveraged jointly with multi-user diversity taking advantage of random differences in users' large and small-scale fading channel parameters with respect to antennas and beams.

Within cells, i.e. intra-cell, MIMO can be used to control interference. Downlink MU-MIMO strategies such as Linear Zero Forced Beamforming use spatial dimensions to send simultaneous transmissions from an array of $N_{T}$ antennas to multiple users so that each receiver (user) sees only its intended signal. Such strategies require full channel state information (FCSI), e.g. a gain and phase value defining the channel between each transmitting antenna and each scheduled user antennas. Furthermore, FCSI would typically also be required from all users considered for scheduling, not just those scheduled. Therefore, in such systems the channel state information (CSI) overheads can be quite large.

Intra-cell strategies can be used within each cell. However, without coordination between cells inter-cell interference (ICI) can severely limit performance. By sharing inter-cell information and coordinating transmissions across cells one can improve performance. In fact, in the fully coordinated case where FCSI from all antennas to all users is known, and transmissions to any user can use any (or all) transmitting antennas, performance can be significantly improved [1], [4]. However, deploying such a system poses significant challenges.

The paper focuses on practical multi-cell systems. Here antennas in a cell transmit only to users in this cell, and transmissions from antennas from neighboring cells are seen as interference to such users. However, we allow base-stations (BSs) to adjust transmissions based on limited information from adjacent cells. Random beamforming is considered [5], [6] since it requires only effective signal powers with respect to each beam, not FCSI. How such a partially coordinated, strictly cellular system, improves over an independent system is of practical interest.

The work in [7] is along such lines, looking at cases where the channel state between a user and its BS are correlated and change slowly with respect to scheduling. Here a BS can learn roughly the channel of its users, and schedule appropriate beams [8]. Furthermore clusters of BSs can jointly schedule beams to help control ICI [7]. In this paper we consider channels that are uncorrelated and independent on each transmission slot. Cells do not act as clusters, though cells are able to infer ICI through beam pilots. We also explore the additional benefits a minimal local exchange of CSI may have over a fully random beamforming approach.

In this paper the focus is on settings involving moderate numbers of users with approaches that combine fair userselection with random beamforming. We explore such approaches and scenarios by simulations. Simplifications to the scenarios could allow for some theoretical analysis but would miss some important effects. However, it should be noted that theoretical analysis of multi-cell scenarios with users clustered at cell-edges can be found in [10]. Capacity growth in the limits of large numbers of users can be found in [11].

The paper is organized as follows. Section II describes the system model and the proportionally fair scheduling approach used. Section III describes various random beamforming strategies for multi-cell downlinks. The schemes address inter- 


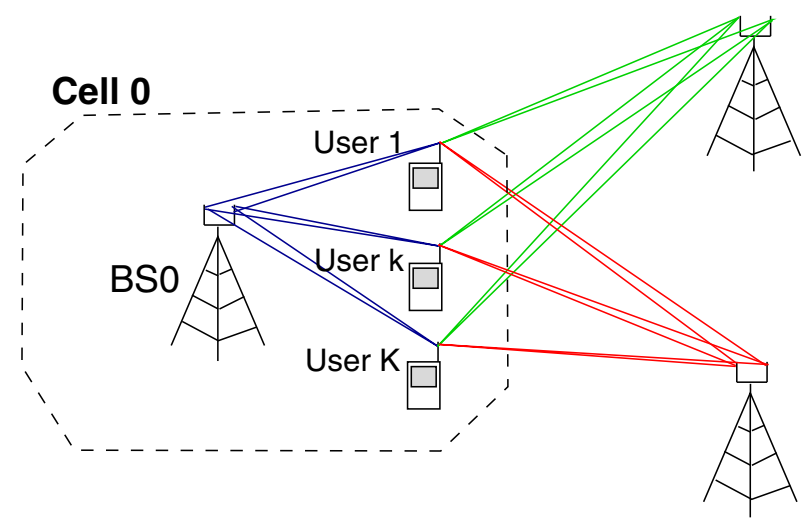

Fig. 1. A cell (cell 0) in a multi-cell multi-antenna downlink system. Here $K$ users are severed by BS 0 . Users receive interference from other cells.

cell interference with different levels of coordination, ranging from a fully independent cell schemes, to a new multi-stage " $A B C$ " scheme that leverages some small patterned (direct) inter-cell FCSI exchanges. Results are presented in Section IV and Section V concludes the paper.

\section{SySTEM MODEL}

As depicted in Fig. 1, we consider a multi-cell downlink system, in which there are $N_{B}$ basestations (BSs) each equipped with $N_{T}$ transmit antennas. The system serves a total of $N_{U}$ users, with each user equipped with a single receive antenna. Each user is assigned to a single basestation in the cellular network. Without loss of generality, we focus on the operation of a particular cell, i.e., cell 0 , which is assigned $K$ users. We assume that $K \gg N_{T}$ to account for the typical operation scenario of next-generation packet-based cellular networks.

The received signal $y_{k}(t)$ of the $k$-th user (in cell 0 ) at time slot $t$ is described as

$$
y_{k}(t)=\sum_{i=0}^{N_{B}-1} \mathbf{h}_{k, i}^{T}(t) \mathbf{x}_{i}(t)+n_{k}(t),
$$

where $\mathbf{x}_{i}(t) \in \mathbb{C}^{N_{T} \times 1}$ is the vector of transmitted signals from the $i$-th $\mathrm{BS}$ at time slot $t, n_{k}(t)$ is independent and identically distributed (i.i.d) circularly symmetric zero-mean unit-power Gaussian noise, and $\mathbf{h}_{k, i}(t) \in \mathbb{C}^{N_{T} \times 1}$ is the channel vector from the $i$-th $\mathrm{BS}$ to the $k$-th user in cell 0 taking into account the effects of path loss, shadowing and Rayleigh fading. We assume a quasi-static (block) fading model, i.e. $\mathbf{h}_{k, i}$ remains constant during scheduling and transmission intervals. Note that we often omit the cell index 0 to simplify notation.

We assume linear precoding and that $m_{i}$ data streams at the $i$-th BS are spatially multiplexed and sent to $m_{i}$ users in the cell. The transmitted signal by the $i$-th $\mathrm{BS}$ is given by

$$
\mathbf{x}_{i}(t)=\sum_{j=1}^{m_{i}} \mathbf{q}_{j, i} s_{j, i},
$$

where $\mathbf{q}_{j, i} \in \mathbb{C}^{N_{T} \times 1}$ is the beam vector assigned to $s_{j, i}$, the $j$-th stream at the $i$-th BS. The model assumes $\left\|\mathbf{q}_{j, i}\right\|=1$ and $\left\|s_{j, i}\right\|^{2}=1 / m_{i} \forall i, j$, i.e. unit norm beams and equal power to users. A transmit power constraint is implicitly included into a gain term applied to $\mathbf{h}_{k, i}$ as described in Section IV. With unit-variance noise this also sets the signal-to-noise ratio.

\section{A. SINR, CSI, CQI and Rate}

Assume that beam $\mathbf{q}_{j, 0}$ is assigned to user $k$ in cell 0 . The signal to interference and noise ratio (SINR) with the $j$-th beam at the $k$-th receiver (user) is

$$
\gamma(k, j)=\frac{\left|\mathbf{h}_{k, 0}^{T} \mathbf{q}_{j, 0}\right|^{2}}{m_{0}} /\left(1+\sum_{\left(j^{\prime}, i^{\prime}\right) \neq(j, 0)} \frac{\left|\mathbf{h}_{k, i^{\prime}}^{T} \mathbf{q}_{j^{\prime}, i^{\prime}}\right|^{2}}{m_{i^{\prime}}}\right)
$$

Assuming Gaussian encoding, the (instantaneous) achievable rate for user $k$ is

$$
R_{k}=\log _{2}[1+\gamma(k, j)] \text {. }
$$

The interference term in (3) can be decomposed into

$$
\sum_{j^{\prime} \neq j} \frac{1}{m_{0}}\left|\mathbf{h}_{k, 0}^{T} \mathbf{q}_{j^{\prime}, 0}\right|^{2}+\sum_{i^{\prime} \neq 0, j^{\prime}} \frac{1}{m_{i^{\prime}}}\left|\mathbf{h}_{k, i^{\prime}}^{T} \mathbf{q}_{j^{\prime}, i^{\prime}}\right|^{2},
$$

The first term comes from inside cell 0 and is intra-cell interference, and the second term comes from other cells and is inter-cell interference. If cell $i$ uses one beam (schedules $m_{i}=1$ user) in a time slot then intra-cell interference is zero for cell $i$. Inter-cell interference is zero for an isolated cell.

The rate given by (4) does not require the full channel state information (FCSI), i.e. it does not require each complex valued entry of vectors $\mathbf{h}_{k, i}$. The rate given by (4) depends on scalars of the form $\left|\mathbf{h}_{k, i}^{T} \mathbf{q}_{j, i}\right|^{2}$. We refer to these scalars as channel quality information (CQI). In random beamforming strategies, each user sends back only the CQI, and not the FCSI to the transmitter. Hence, the feedback overhead of random beamforming strategies is much less compared to schemes such as LZF when the number of users is large.

\section{B. Proportional Fair Scheduling}

A scheduler is employed to choose the set of users served in each slot and the rate allocated to each user in that slot. We consider the multiuser proportional fair scheduling (MPFS) algorithm [9] for multi-cell environments.

For cell 0 , let $\mathbb{U}(n)$ be the set of users scheduled at slot $n$, and $R_{k}(n, \mathbb{U}(n))$ the rate scheduled to user $k$ at slot $n$. The average throughput of user $k$ up to (and including) slot $n$ is denoted as $T_{k}(n)$ and is updated as follows,

$$
T_{k}(n)=\left(1-\frac{1}{\tau}\right) T_{k}(n-1)+\frac{1}{\tau} R_{k}(n, \mathbb{U}(n)),
$$

where $\tau$ is a parameter related to the time interval over which fairness is achieved. Note that $R_{k}(n, \mathbb{U}(n))=0$ if user $k$ in cell 0 is not scheduled at time slot $n$.

For the case $\tau \gg 1$ the system is a PFS system that tries to maximize the total utility function defined by $\sum_{k} \log _{2}\left(T_{k}(n)\right)$. It schedules a set of users $\mathbb{U}(n)$ in cell 0 according to

$$
\arg \max _{\mathbb{U}(n)} \sum_{k \in \mathbb{U}(n)} \frac{R_{k}(n, \mathbb{U}(n))}{T_{k}(n-1)} .
$$

At time slot $n$, the PFS scheduler (in cell 0) uses the windowed average rate $T_{k}(n-1)$ as a weight for each user $k$, and finds the user subset $\mathbb{U}(n)$ and the appropriate user rates to maximize the weighted sum rate defined by (7).

\section{Multi-Cell Random Beamforming Strategies}

The goal is to manage inter-cell interference with limited channel information feedback and inter-cell exchange. We study and compare three classes of strategies with differing requirements in feedback and (inter-cell) coordination. 


\section{A. Baseline Independent Cell Schemes}

In all base-line systems, each cell operates as an isolater cell and there is no exchange or use of inter-cell informatio In the first base-line system, the Fully Independent system, a cells operate at the same time with frequency reuse one. Eac cell constructs random beamforming vectors, schedules, ar transmits to users independently and simultaneously ignorin (assuming zero) interference from other cells.

The second base-line system, a "TDM" system, an extension of this scheme based on time divisic or frequency reuse. The cells are broken down in three subsets, "A" $=\left\{a_{1}, \ldots, a_{n_{A}}\right\}$, "B" $=\left\{b_{1}, \ldots, b_{n_{B}}\right\}$, ar "C" $=\left\{c_{1}, \ldots, c_{n_{C}}\right\}$ as in a cellular frequency reuse patter for reuse 3. See Fig. 2. With reuse one, in the TDM schen only one subset operates at a time (or equivalently differes subsets use different frequencies). Therefore there is no inte subset interference, and cells operate independently ignoring interference from all other cells within the same subset. Here the assumption is that interference between cells in the same subset is small and can be ignored. In reality, as shown later, even for the TDM scheme, ICI is non-negligible.

In either of these schemes at each transmission slot the BS for cell $i$ selects $m_{i}$ beams at random in an i.i.d. fashion. In these systems, each user needs only to estimate the CQI of beams from its assigned BS and feedback the CQI only to that BS. It does not need to monitor the CQI with respect to, or send feedback to, other BSs. If multiple users are scheduled in a cell, intra-cell interference is known through the CQI of scheduled users and accounted for. For selecting users intercell interference is ignored. Each BS selects its users via (7), with $R_{k}$ given by (4) and $\gamma(k, j)$ by (3), and where the ICI term, i.e., the second term in (5), is assumed to be zero ${ }^{1}$.

\section{B. SINR: Joint Multi-Cell User-Scheduling by SINR Feedback}

In this scheme, users are selected based on SINR levels using information about known inter-cell interference. As in baselines schemes FCSI is not necessary. Furthermore, CQI information about inter-cell interference is not shared directly between base-stations. Rather, information is exchanged indirectly by users sensing the beam-pilots from adjacent cells.

In the "SINR" scheme each BS selects $m$ beam vectors for its cell randomly and independently. Each BS then sends $m$ beam-pilots with which users estimate CQI. A user in a given cell estimates not only the CQI with respect to beams of its BS, but also the CQI of beams it can sense from neighboring $\mathrm{BSs}^{2}$. Each user then selects a beam for signalling (the beam with maximum CQI) from its BS. Given this selection, it calculates the SINR based on the other sensed beams from both its own and other BSs according to (3). The user then responds to

\footnotetext{
${ }^{1}$ Once users have been scheduled on beams in a baseline system, the BSs do not know the inter-cell interference terms in (3), and therefore cannot compute the actual achievable rate (4). For comparison purposes, however, we use the actual achievable rate (4) with the interference given by (3) as an (optimistic) benchmark of the rates that can achieved by a baseline scheme.

${ }^{2}$ Pilots are sent in a way that a user is able to sense all pilots.
}

its BS with the selected signalling beam's index and a rate request based on (4) given the calculated SINR.

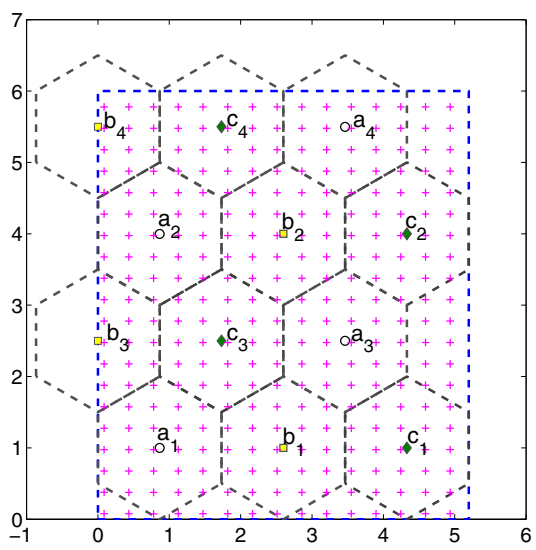

Fig. 2. Simulation layout: there are 12 cells separated by 1 unit on a topology wrapped to form a flat $2 \mathrm{D}$ torus. The $N_{U}$ users are drawn from a uniform distribution on a $15 \times 20$ grid (each grid point might have multiple users). Cell assignments for one slot are shown using labels $a_{i}, b_{i}$ and $c_{i}$.

After receiving all the rate requests and beam choices from its users, a BS selects the users for each beam based on (7) and transmits at the achievable rate. The system leverages userselection to select users for which the useful signal is strong and interference is small due to pathloss/fading. ${ }^{3}$

\section{ABC: Joint Multi-Cell Scheduling and Beam Coordination}

The ABC scheme improves performance using a practical distributed scheduling algorithm that manages interference via both user selection and inter-cell dependent beam selection. The scheme partitions a network of cells into $\mathrm{A} / \mathrm{B} / \mathrm{C}$ subsets as in Fig. 2, but using frequency reuse 1. As in the "SINR" scheme, it leverages indirect information exchange by using beam-pilots in adjacent cells. It also allows for some user selections to be made ahead of some beam selections, allowing beam coordination to reduce inter-cell interference.

The scheme starts on the first slot with cells divided into three disjoint subsets "A" $=\left\{a_{1}, \ldots, a_{n_{A}}\right\}$, "B" $=\left\{b_{1}, \ldots, b_{n_{B}}\right\}$, and "C" $=\left\{c_{1}, \ldots, c_{n_{C}}\right\}$ as in Fig. 2 . With this the first transmission slot consists of the following four steps:

\section{- Step 1 (Beam and User Scheduling for A cells):}

Each $a_{i} \in$ A selects $m$ beams $\left\{\mathbf{q}_{1, a_{i}}, \ldots, \mathbf{q}_{m, a_{i}}\right\}$ and schedules $m$ users $\left\{u_{k_{1}, a_{i}}, \ldots, u_{k_{m}, a_{i}}\right\}$ independently of other $\mathrm{A}$, $\mathrm{B}$ and $\mathrm{C}$ cells as in baseline schemes. B and C-cell users sense A-cell beam-pilots during this process.

\section{- Step 2 (Beam and User Scheduling for B cells):}

Each $a_{i} \in \mathrm{A}$ sends to a subset of B-cells, $\mathbb{B}_{a_{i}} \subset B$, the identity of users $\left\{u_{k_{1}, a_{i}}, \ldots, u_{k_{m}, a_{i}}\right\}$. In simulations $\mathbb{B}_{a_{i}}$ are the Bcells adjacent to $a_{i}$. Based on this, and FCSI of those users, each $b_{i} \in$ B selects beams $\left\{\mathbf{q}_{1, b_{i}}, \ldots, \mathbf{q}_{m, b_{i}}\right\}$ that limit interference to neighboring A cell scheduled users.

Each $b_{i} \in \mathrm{B}$ schedules $m$ users $\left\{u_{k_{1}, b_{i}}, \ldots, u_{k_{m}, b_{i}}\right\}$ independently of $\mathrm{C}$ cells and other B cells. It does so using SINR

\footnotetext{
${ }^{3}$ It is assumed that all cells use all the beams for which beam-pilots are sent and that beams have the same power level (or the power level is implicit in the pilot). This way inter-cell interference can be estimated by users.
} 
feedback (as in the SINR scheme) given known $\mathrm{A} \rightarrow \mathrm{B}$ intercell interference its users obtained from A-Cell pilots in Step 1. C-Cell users sense B-Cell beam-pilots during this process.

- Step 3 (Beam and User Scheduling for $\mathbf{C}$ cells):

Each A and B cell sends as above (limited) information of its scheduled users to a subset of (neighboring) $\mathrm{C}$ cells. Based on this each $\mathrm{C}$ cell selects beams in order to limit interference to neighboring $\mathrm{A}$ and $\mathrm{B}$ cell users.

Then, each C cell selects users independently of other C-Cells, using SINR feedback given known $\mathrm{A} \rightarrow \mathrm{C}$ and $\mathrm{B} \rightarrow \mathrm{C}$ inter-cell interference its users obtained from A and B-Cell beam-pilots.

- Step 4 (All cells transmit together): All cells from subsets

$\mathrm{A}, \mathrm{B}$ and $\mathrm{C}$ transmit given the beam and user selections above. It can be shown that users in the A-cells have advantages over (tend to get larger rates than) B and C-cell users. Similarly B-Cell users have advantages over C-cell users. To equalize rates achieved in cells, a final Step 5 changes cell assignments in a round-robin fashion before the next transmission slot:

- Step 5 (Shuffle Assignment): Assign present A-cells to set B, present B-cells to set C, present C-cells to A. Go to Step 1 .

Figure 3 shows the flow of the system. Components of these steps are now described in more detail.

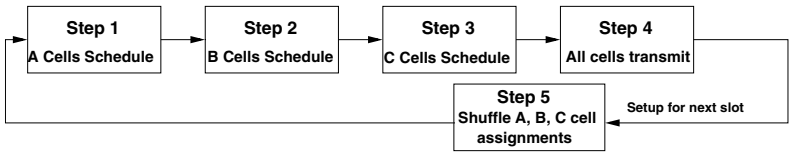

Fig. 3. Flow diagram of steps in $\mathrm{ABC}$ scheme

\section{III.C.1. Limited Inter-cell Information Passing}

In step 2, each $b_{i} \in \mathrm{B}$ knows the users scheduled in a subset of (the adjacent 3) A-cells. Assume that the three closest Acell neighbors are considered, with $m$ users per cell. Label these $3 m$ A-cell users, $\left\{x_{k_{1}, b_{i}}, \ldots, x_{k_{3 m}, b_{i}}\right\}$, where $x_{k_{1}, b_{i}}=$ $u_{k_{j}, a(p)}$ for some $j$ and $p$. The B-cell sends out channel-pilots with which these users estimate the FCSI with respect to the B-cell. This FCSI can be sent directly from A users to the B cell station, or forwarded from A cells. The collected FCSI forms a $\mathcal{C}^{N_{t} \times(3 m)}$ matrix

$$
\mathbf{H}_{b_{i}}=\left[H_{x_{k_{1}, b_{i}} \leftarrow b_{i}}, \cdots, H_{x_{k_{3 m}, b_{i}} \leftarrow b_{i}}\right]
$$

where $H_{x_{k_{j}, b_{i}} \leftarrow b_{i}}$ is the FCSI of user $x_{k_{j}, b_{i}}$ with respect to the $N_{T}$ antennas of $b_{i}$. Similarly in step 3 , if each C-cell considers three A-cells and three B-cells the C-Cell considers a $\mathcal{C}^{N_{t} \times(6 m)}$ FCSI matrix for the scheduled A and B cell users.

\section{III.C.2. Beam Coordination}

To choose random beams in Steps 2 and 3 we consider a simple criterion to minimize the sum interference to the users previously selected in other cells. Specifically, cell $b_{i}$ calculates the eigenvalue decomposition of $\mathbf{H}_{b_{i}} \mathbf{H}_{b_{i}}^{H}$, denoted by $U_{b_{i}} \Sigma_{b_{i}} U_{b_{i}}^{H}=\mathbf{H}_{b_{i}} \mathbf{H}_{b_{i}}^{H}$. It selects the $m$ beam vectors used in cell $b_{i}$ as the eigenvectors (columns of $U_{b_{i}}$ ) corresponding to the $m$ minimum eigenvalues. $\mathrm{C}$-cells operate similarly.

\section{Simulation Results}

We present performance comparisons of various schemes from the perspective of per-user and per-cell throughput, based on simulations conducted on the 12-station cellular network shown in Fig. 2. We assume that each BS is located at the center of a hexagonal cell, with BS's separated by 1 unit. A flat torus (wrapped geography) topology is used with respect to the dashed rectangle to avoid the boundary effects. Each basestation is equipped with $N_{T}$ antennas and each user with one antenna. The $N_{U}$ users are drawn from a uniform distribution on a $15 \times 20$ grid. Therefore there are $N_{U} / 300$ users on average located at each grid point and $N_{U} / 12$ users on average per hexagonal cell. In all systems $m_{i}=m \forall i$. The MPFS algorithm is run with $\tau=500$ and $T_{k}(n)$ and is made to converge before noting rate statistics.

The channel model we employ accounts for path loss, lognormal shadow fading and Rayleigh fading. Every user is assigned to a basestation with the highest average received signal strength depending on path loss and shadowing. The combined effect of a transmit power $P$ and path loss at a distance $d$ from a base-station is given by

$$
P L(d)=\left(G_{0} / d_{0}^{\alpha}\right)\left[1+\left(d / d_{0}\right)^{\alpha}\right]^{-1}
$$

The settings used are $\alpha=3.76, d_{0}=\sqrt{2} / 10$ and $G_{0}=$ 12.94. Thus, accounting only for path loss, nominal SNRs at the edges of the cells are between 16.8 and $19.1 \mathrm{~dB}$, while at the cell center the SNR is $43.1 \mathrm{~dB}$. SINR values however can be significantly less. Log-normal shadowing is considered with zero mean and a shadowing standard deviation $\sigma=8$ dB. Rayleigh fading components are i.i.d. complex Gaussian variables with zero mean and unit variance. With the power constraint (gain) included into $\mathbf{h}_{k, i}$ 's through (9), all beams are of unit norm and $\left\|s_{j, i}\right\|^{2}=1 / m$ for $\forall i, j=1, \ldots, m$.

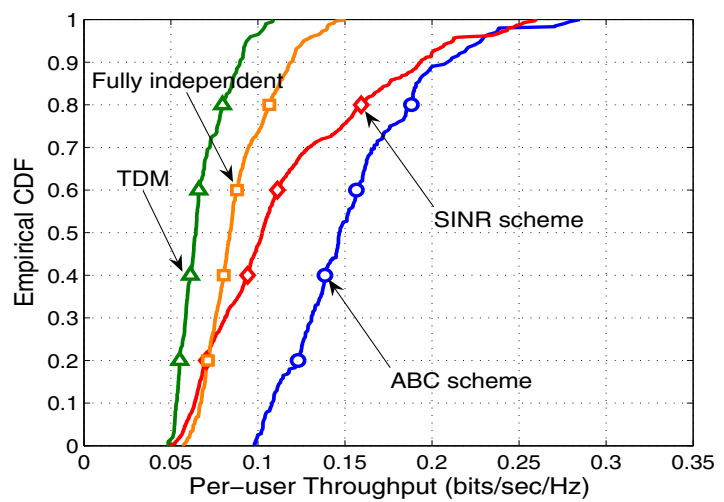

Fig. 4. Empirical CDF of the per-user throughputs over the 12-base cellular system, with $N_{T}=6$ and 50 users per cell on average, and $m=1$ user/cell.

Fig. 4 shows the empirical CDF of per-user throughput for different schemes when $N_{T}=6, m=1$ and the number of users per cell is 50 on average $\left(N_{U}=600\right)$. The TDM scheme, which avoids strong interference by dividing resources, performs the worst among the tested schemes. The fully independent cell scheduling scheme, with frequency reuse 1 , increases per-user throughput despite the fact it does not have an interference management mechanism. The multi-cell SINR feedback scheme increases the throughput performance significantly for users relatively close to BSs, mitigating interference via user selection. The ABC scheme, with 


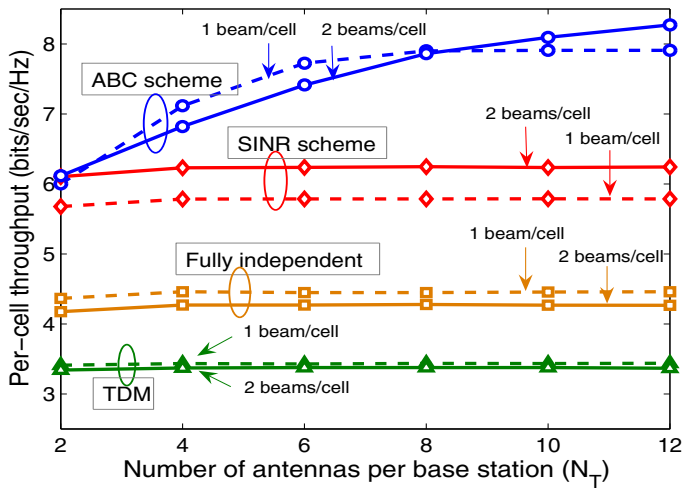

Fig. 5. Average per-cell throughput of different schemes versus $N_{T}$.

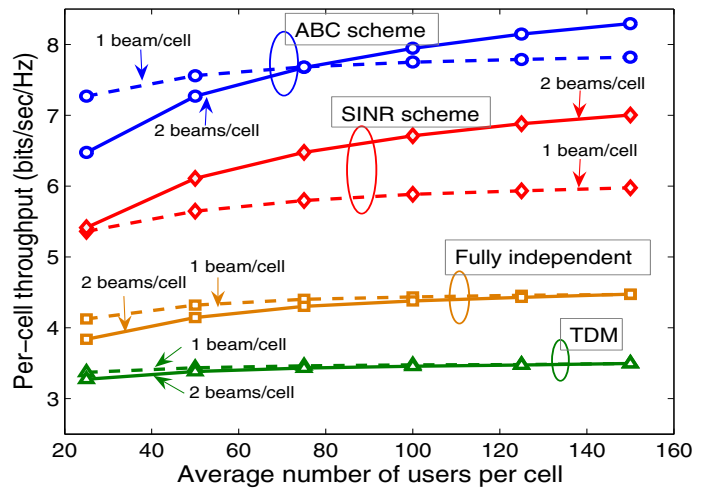

Fig. 6. Average per-cell throughput of different schemes versus average number of users per cell $\left(N_{U} / 12\right)$, for $N_{T}=6$.

added coordination, obtains the best throughput performance, especially for edge users (edge rates of the $\mathrm{ABC}$ scheme are about twice those of other schemes). It shows that added intercell beam coordination has strong advantages.

In Fig. 5, we show the average per-cell sum-throughput for $N_{U}=600$ of different schemes versus $N_{T}$ for $m=1$ and $m=2$. Note, increasing $m$ increases intra-cell interference which can decrease rates for schemes without any interference avoidance mechanism. This is seen in baseline schemes. Also note, while the nomimal level of inter-cell interference does not change with $m$, since we constrain the sum power as described in Section II, more spatial dimensions are illuminated with increasing $m$. In fact if $6 m \geq N_{T}$ the $\mathrm{ABC}$ scheme can not select beams to fully avoid interference between adjacent cells.

When using random beams increasing the number of antennas (the dimension of the space) can help the system find users with lower the interference terms, i.e. users with lower " $\left|\mathbf{h}_{k, i}^{T} \mathbf{q}_{j^{\prime}, i}\right|$ " in (5). Under a moderate number of users per cell (50 user in Fig. 5), the net benefit to the SINR scheme is negligible. Nonetheless, the scheme itself is able to schedule effectively $m=2$ users per cell even with $N_{T}=2$. The $\mathrm{ABC}$ scheme, with non-random beam selection, does leverage increased $N_{T}$, outperforming other schemes even with $m=1$.

Fig. 6 shows the dependence on the number of users per cell when $N_{T}=6$. The benefits of increased $N_{U}$ to user-selection in the SINR and ABC schemes are clearly seen. When the number of users per cell is small, higher throughput can be obtained by using 1 beam per cell. However, when the number of users per cell increases, additional beams can be scheduled in each cell since there is a higher probability that there exists a user whose channel vector is near-orthogonal to the previous scheduled beam(s), and scheduling additional beam does not introduce much interference.

\section{CONCLUSION}

In this paper we investigated various limited coordination random beamforming strategies for multi-cell downlink communication. The SINR scheme requires only CQI information, obtained by using beam-pilots without direct BS-toBS exchanges. Direct BS-to-BS exchanges are used only for the ABC scheme, and consists of FCSI for a small number of scheduled users. Such constraints do limit performance compared to techniques such as LZF. Still, the SINR scheme using CQI and user selection only can deliver more than twice the throughput of fully independent-cell schemes. This is significant given the practical simplicity of the scheme.

The results also suggest that with moderate numbers of users random beamforming does have limits in leveraging extra spatial dimensions to manage interference. This is suggested by similar performance at times between 1 and 2 beam/cell cases and the slow growth of performance with $N_{T}$ when FCSI is not used. In fact, we investigated other schemes where, in ABC-like patterns, cells are assigned 1 or 2 beams/cell with similar results. Cluster based beam selections in [7] also show marginal improvements. This suggests that some intercell CSI exchange, as in the $\mathrm{ABC}$ scheme, may be required to effectively leverage larger $N_{T}$ to mitigate ICI and take more advantage of the spatial dimensions introduced by MIMO.

\section{REFERENCES}

[1] M. K. Karakayali, G. J. Foschini, and R. Valenzuela, "Network coordination for spectrally efficient communications in cellular systems," IEEE Wireless Commun., vol. 13, no. 4, pp. 56-61, Aug. 2006.

[2] A. Goldsmith, S.A. Jafar, N. Jindal, and S. Vishwanath, "Capacity limits of MIMO channels," IEEE J. on Select. Areas in Commun., vol. 21, no. 5, pp. 684-702, Jun. 2003.

[3] D. Gesbert, M. Kountouris, R. Heath, C. B. Chae, and T. Salzer, "From single user to multiuser communications: shifting the MIMO pradigm," IEEE Sig. Proc. Mag., vol. 24, no. 5, pp. 36-46, Sep. 2007.

[4] S. Shamai and B. M. Zaidel, "Enhancing the cellular downlink capacity via co-processing at the transmitting end," in Proc. IEEE Veh. Tech. Conf., Rhodes, Greece, May 2001, pp. 1745-1749.

[5] M. Sharif and B. Hassibi, "On the capacity of MIMO broadcast channels with partial side information," IEEE Trans. Inf. Theory, vol. 51, no. 2, pp. 506-522, Feb. 2005.

[6] P. Viswanath, D.N.C. Tse, and R. Laroia, "Opportunistic beamforming using dumb antennas," IEEE Trans. Inf. Theory, vol. 48, no. 6, pp. 12771294, Jun. 2002.

[7] J. Ling M. Vemula, D. Avidor and C. Papadias, "Inter-cell coordination, opportunistic beamforming and scheduling," in Proc. ICC, Istanbul, Turkey, June 2006.

[8] J. Ling D. Avidor and C. Papadias, "Jointly opportunistic beamforming and scheduling (JOBS) for downlink packet access," in Proc. ICC, Paris, France, June 2004.

[9] M. Kountouris and D. Gesbert, "Memory-based opportunistic multi-user beamforming," in Proc. Int. Symp. Inf. Theory, Adelade, Aust., Sep. 2005.

[10] S. Jing, D. N. C. Tse, J. Hou, J. B. Soriaga, J. E. Smee and R. Padovani, "Multi-Cell Downlink Capacity with Coordinated Processing," in Proc. ITA'07, San Diego, CA, Feb. 2007

[11] D. Gesbert and M. Kountouris, " Resource allocation in multicell wireless networks: Some capacity scaling laws," in Proc. of WiOpt 2007, Limassol, Cyprus, Apr. 2007 